\title{
Biomimetic formation of vaterite mesocrystals
}

\author{
QI-ZHI YAO ${ }^{1}$, TIAN-LEI ZHAO ${ }^{2}$, GEN-TAO ZHOU ${ }^{2}$
}

${ }^{1}$ School of Chemistry and Materials Science, University of Science and Technology of China, Hefei, 230026. qzyao@ustc.edu.cn.

${ }^{2}$ School of Earth and Space Sciences, University of Science and Technology of China, Hefei, 230026.

The distinctive feature of biomineralization is that organisms can precisely control crystallization and morphogenesis of minerals. Diverse organisms have the ability to tailor the polymorph and architecture of $\mathrm{CaCO}_{3}$ for their natural needs. Vaterite is the least stable polymorph of anhydrous calcium carbonate, but biogenetic vaterite exhibits high stability compared to its abiotic counterpart. Therefore, biogenetic vaterite widely occurs in freshwater cultured pearls, larval snail, fish otoliths, and some inorganic tissues like gallstones and human heart valves, and the vaterite usually grows into complex habits, such as tablets, fibers, and botryoidal habits with lamellar superstructures. For the vaterite, a consensus is that polyanionic macromolecules, such as proteins and polysaccharides, can induce the nucleation of special polymorph and control the unique morphogenesis with their carboxylate, sulfate, and phosphate groups. Here, a biomimetic mineralization technique was used to investigate the effect of model additives sodium citrate and sodium dodecyl benzene sulfonate on crystallization and growth of $\mathrm{CaCO}_{3}$. A series of results show that vaterite prisms consisting of well-stacked nanoflakes can be achieved in the presence of the additives. The TEM, SAED and BET analyses confirm that the prismatic architectures are orientationally aligned mesocrystals of vatreite. The control experiments further reveals that the vaterite mesocrystal formation can be attributed to the cooperative interactions between citrate and dodecyl benzene sulfonate. The driving force controlling the self-assembly of the nanoflake subunits into mesocrystals may originate from the van der Waals force and/or hydrogen bonds of the adsorbed/anchored organic molecules on the subunits and the inherent anisotropic dipole-dipole interactions between the assembled subunit crystals.

This work was financially supported by the Natural Science Foundation of China (Nos. 41572026 and 41772030). 